\title{
Detection of Gauss-Markov Random Fields With Nearest-Neighbor Dependency
}

\author{
Animashree Anandkumar, Student Member, IEEE, Lang Tong, Fellow, IEEE, and Ananthram Swami, Fellow, IEEE
}

\begin{abstract}
The problem of hypothesis testing against independence for a Gauss-Markov random field (GMRF) is analyzed. Assuming an acyclic dependency graph, an expression for the log-likelihood ratio of detection is derived. Assuming random placement of nodes over a large region according to the Poisson or uniform distribution and nearest-neighbor dependency graph, the error exponent of the Neyman-Pearson detector is derived using large-deviations theory. The error exponent is expressed as a dependency-graph functional and the limit is evaluated through a special law of large numbers for stabilizing graph functionals. The exponent is analyzed for different values of the variance ratio and correlation. It is found that a more correlated GMRF has a higher exponent at low values of the variance ratio whereas the situation is reversed at high values of the variance ratio.
\end{abstract}

Index Terms-Detection and estimation, error exponent, Gauss-Markov random fields, law of large numbers.

\section{INTRODUCTION}

$\mathbf{F}$ OR distributed detection, the so-called conditionally independent and identically distributed (i.i.d.) assumption is mathematically convenient and is widely assumed in the literature. The assumption states that conditioned on a particular hypothesis, the observations at sensors are independent and identically distributed. In practice, however, spatially distributed sensors often observe correlated data, since natural spatial signals have stochastic dependence. Examples of correlated signals include measurements from temperature and humidity sensors, or from magnetometric sensors tracking a moving vehicle. Audio data is also rich in spatial correlations, due to the presence of echoes.

Spatial random signals are typically acausal in contrast to temporal signals. In the literature, the two are usually distinguished by referring to acausal signals as random fields (RF) and causal signals as random processes (RP). Random fields are

Manuscript received January 02, 2007; revised January 31, 2008. Current version published February 04, 2009. This work was supported in part through the collaborative participation in the Communications and Networks Consortium sponsored by the U.S. Army Research Laboratory under the Collaborative Technology Alliance Program, Cooperative Agreement DAAD19-01-2-0011 and by the Army Research Office under Grant ARO-W911NF-06-1-0346. The U.S. Government is authorized to reproduce and distribute reprints for Government purposes notwithstanding any copyright notation thereon. The material in this paper was presented in part at IEEE International Conference on Acoustics, Speech and Signal Processing, Hawaii, April 2007.

A. Anandkumar and L. Tong are with the School of Electrical and Computer Engineering, Cornell University, Ithaca, NY 14853 USA (e-mail: aa332@ece. cornell.edu; ltong@ece.cornell.edu).

A. Swami is with the Army Research Laboratory, Adelphi, MD 20783 USA (e-mail: a.swami@ieee.org).

Communicated by A. Høst-Madsen, Associate Editor for Detection and Estimation.

Color versions of Figures 1-7 in this paper are available online at http://ieeexplore.ieee.org.

Digital Object Identifier 10.1109/TIT.2008.2009855

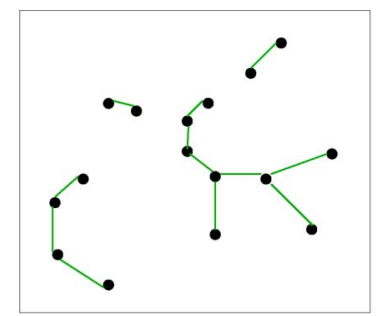

(a) $\mathcal{H}_{1}$ : Gauss-Markov random field with nearest-neighbor dependency.

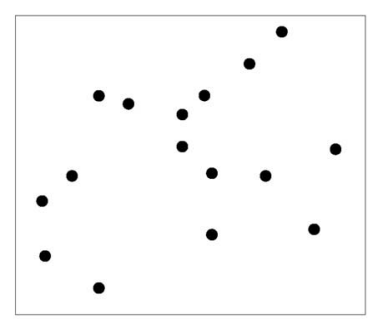

(b) $\mathcal{H}_{0}:$ Independent observations
Fig. 1. Illustration of the hypothesis-testing problem.

of interest in a variety of engineering areas and may represent natural phenomena such as the dispersion of atmospheric pollutants, groundwater flow, rainfall distribution or the mesoscale circulation of ocean fields [2].

In this paper, we consider the problem of hypothesis testing for independence, shown in Fig. 1. Specifically, under the alternative hypothesis, sensors collect samples from a Gauss-Markov random field (GMRF), whereas the samples are independent under the null hypothesis. We model the GMRF through a graphical approach, in which a dependency graph (DG) specifies the stochastic dependence between different sensor observations. This dependency graph can have different degrees of sparsity and can even be fully connected. However typically, spatial interactions are based on proximity, where the edges are included according to some specified rule based on the local point configuration [3], [4]. With a regular lattice structure ( e.g., in image processing, Ising model), a fixed set of neighbors can be specified in a straight-forward manner [5]. However, the situation is more complicated for arbitrarily placed nodes. In this paper, we consider the nearest-neighbor graph (NNG), which is the simplest proximity graph. The nearest-neighbor relation has been used in several areas of applied science, including the social sciences, geography and ecology, where proximity data is often important [6], [7].

We consider the Neyman-Pearson (NP) formulation, where the detector is optimal at a fixed false-alarm probability. But, under this formulation, analysis of performance metrics such as error probability is intractable for an arbitrary number of observations. Hence, we focus on the large-network scenario, where the number of observations goes to infinity. For any positive fixed level of false alarm or the type-I error probability, when the misdetection or the type-II error probability $P_{M}(n)$ of the NP detector decays exponentially with the sample size $n$, we have the error exponent defined by

$$
D:=-\lim _{n \rightarrow \infty} \frac{1}{n} \log P_{M}(n) .
$$


The error exponent is an important performance measure since a large exponent implies faster decay of error probability with increasing sample size.

Additionally, we assume that the sensors observing the signal field are placed i.i.d. according to the uniform or Poisson distribution. Since nodes are placed irregularly, it results in a non-stationary GMRF (for the definition of stationary GMRF, see [8, p. 57]). We assume that the number of nodes goes to infinity, by way of the coverage area of the nodes going to infinity, while keeping the node density fixed. Under this formulation, we derive the detection error exponent, assuming access to all the observations.

\section{A. Related Work and Contributions}

The kind of hypothesis testing we consider is called testing for independence. In [9] and [10], problems of this kind are considered with rate constraints on the channels and for two sources, using a large number of samples at each source. In this paper, we assume that there are no constraints on the channel and that the observations have the correlation structure of the GMRF. Our formulation is different since there is a single observation at every sensor, and the number of sensors goes to infinity.

GMRF is also known as conditional autoregression (CAR) in the seminal work of Besag [11], [12]. They have a wide array of applications in fields such as speech recognition, natural language processing, coding, geo-statistics, image analysis and AI. The literature is too vast to mention here. For an exposition on GMRF, see [8], [13].

Another related problem is the detection of Gauss-Markov random processes (GMRP) in Gaussian noise, which is a classical problem [14]. There is an extensive literature on the large-deviations approach to the analysis of detection of GMRP [15]-[25], but closed-form expressions have been derived only for some special cases, e.g., [26]-[28]. GMRP has been characterized via inversion algorithms for block-banded matrices [29], [30]. However, these approaches are not amenable to the extension of the problem to planar and higher dimensional spaces, since they deal with random processes rather than random fields, or to the random placement of nodes.

Related to the GMRF, there is an alternative and more restrictive approach, known as the spatial auto-regressive model (SAR) and has been extensively studied in the field of spatial data-mining. In [7], this formulation is considered with (directed) nearest-neighbor interaction and a closed-form ML estimator of the AR spatial parameter is characterized. We do not consider this formulation in this paper.

To our knowledge, large-deviation analysis of the detection of acausal nonstationary GMRF has not been treated before. We first express the likelihood function of a GMRF with an arbitrary acyclic dependency graph, in terms of its covariance matrix. The joint distribution can also be derived by expressing it in terms of the marginal probability of the nodes and the joint probability at the edges of the dependency graph [31], [32].

We consider the detection problem represented in Fig. 1, under the additional assumptions of nearest-neighbor dependency. We consider the location of the sensors as a random point set drawn from uniform or Poisson distribution and defined on expanding regions. This framework allows us to exploit recent advances in computational geometry [33], [34]. By casting the error exponent as a limit of the sum of graph functionals, we are able to apply the special law of large numbers (LLN) for functionals on graphs derived in [33]. We obtain the final form of the exponent by exploiting some special properties of the NNG. We then numerically evaluate the exponent for different values of the variance ratio and correlation, for exponential and constant correlation functions. We conclude that at a fixed node density, a more correlated GMRF has a higher exponent at low values of variance ratio, whereas the opposite is true at high values of variance ratio.

\section{B. Notation and Organization}

Vectors and matrices are written in boldface. Random variables are in capital letters, random processes and random fields in boldface capitals and sets in calligraphic font. For the matrix $\mathbf{A}=[A(i, j)], A(i, j)$ denotes the element in the $i$ th row and $j$ th column and $|\mathbf{A}|$ its determinant. For sets $\mathcal{A}$ and $\mathcal{B}$, let $\mathcal{A} \backslash \mathcal{B}=\{i: i \in \mathcal{A}, i \notin \mathcal{B}\}$ and let $|\cdot|$ denote cardinality.

An undirected graph $\mathcal{G}$ is a tuple $\mathcal{G}=(\mathcal{V}, \mathcal{E})$ where $\mathcal{V}=$ $\{1,2, \ldots, n\}$ is the vertex ${ }^{1}$ set and $\mathcal{E}=\{(i, j), i, j \in \mathcal{V}, i \neq j\}$ is the edge set. When $i$ and $j$ have an edge between them, $i$ and $j$ are neighbors denoted by $i \sim j$ (otherwise, it is $i \nsim j$ ). For a directed graph, we denote the edges by $\mathcal{E}=\{\langle i, j\rangle, i, j \in$ $\mathcal{V}, i \neq j\}$, where the direction of the edge is from $i$ to $j$. The neighborhood function of a node $i$ is the set of all other nodes having an edge with it, i.e.

$$
\mathcal{N}(i)=\{j \in \mathcal{V}: j \neq i,(i, j) \in \mathcal{E}\} .
$$

The number of neighbors of a node $i$ is called its degree, denoted by $\operatorname{Deg}(i)$. A node with a single edge i.e., its degree is 1 is known as a leaf and the corresponding edge as a leaf edge, otherwise it is known as an internal or interior edge. Let $\operatorname{dist}(i, j)$ be the Euclidean distance between any two nodes. Let $R_{i j}$ denote the (random) Euclidean edge-length of $(i, j)$ in graph $\mathcal{G}=(\mathcal{V}, \mathcal{E})$

$$
R_{i j}=\operatorname{dist}(i, j), \quad \forall(i, j) \in \mathcal{E} .
$$

Our paper is organized as follows. We provide a description of the GMRF in Section II, focusing on the acyclic dependency graph in Section III and providing an expression for the likelihood function in Section III-A. We define the hypothesistesting problem in Section IV and specify additional assumption on the covariance matrix of the GMRF in Section IV-A. In Section IV-B, we assume additionally that the dependency graph is the nearest-neighbor graph. We provide an expression for the log-likelihood ratio in Section IV-C. We define the error exponent under the Neyman-Pearson formulation in Section V and specify the random placement of nodes in Section V-A. In Section VI we evaluate the error exponent, expressing it as a graph functional in Section VI-A, applying the LLN for graphs in Section VI-B, and providing an explicit form for NNG in Section VI-C. We provide numerical results for the exponent in Section VI-D, and Section VII concludes the paper.

\footnotetext{
${ }^{1}$ We consider the terms node, vertex and sensor interchangeable.
} 


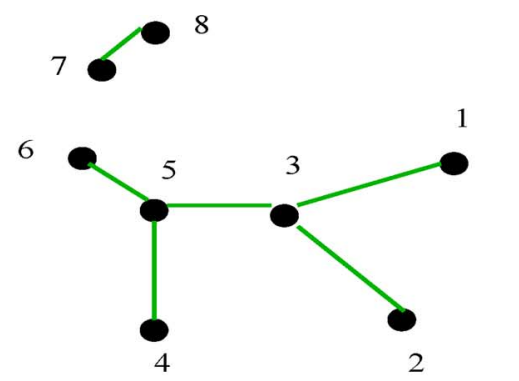

(a) A labeled simple undirected graph.

Fig. 2. Dependency graph and potential matrix of a GMRF.

\section{GAUSS-MARKov RANDOM FIELD}

A GMRF, in addition to being a Gaussian random field, satisfies special conditional independence properties. A simple example is the first-order AR process, where the conditional independence of the observations is based on causality. However, a spatial random field has a far richer set of conditional independencies, requiring a more general definition $[8$, p. 21].

Definition 1 (GMRF): Given a point set $\mathcal{V}=\{1, \ldots, n\}$, $\mathbf{Y}_{\mathcal{V}}=\left\{Y_{i}: i \in \mathcal{V}\right\}$ is a GMRF with an (undirected) dependency graph $\mathcal{G}(\mathcal{V}, \mathcal{E})$ if $\mathbf{Y}_{\mathcal{V}}$ is a Gaussian random field, and $\forall i, j \in \mathcal{V}, Y_{i}$ and $Y_{j}$ are conditionally independent given observations at all other nodes if $i$ and $j$ are not neighbors, i.e.,

$$
Y_{i} \perp Y_{j} \mid \mathbf{Y}_{-i j} \Longleftrightarrow i \nsim j, \forall i, j \in \mathcal{V}, i \neq j
$$

where $\perp$ denotes conditional independence and $\mathbf{Y}_{-i j}:=\left(Y_{k}\right.$ : $k \in \mathcal{V}, k \neq i, j$ ).

A common approach to formulating a GMRF is to specify the dependency graph through a neighborhood rule and then to specify the correlation function between these neighbors. Thus, in a GMRF, local characteristics completely determine the joint distribution of the Gaussian field.

The following Markov properties are equivalent in a GMRF:

1) pairwise-Markov property

$$
Y_{i} \perp Y_{j} \mid \mathbf{Y}_{-i j} \Longleftrightarrow(i, j) \notin \mathcal{E} ;
$$

2) local-Markov property

$$
Y_{i} \perp \mathbf{Y}_{-(i, \mathcal{N}(i))} \mid \mathbf{Y}_{\mathcal{N}(i)}
$$

3) global-Markov property

$$
\mathbf{Y}_{\mathcal{A}} \perp \mathbf{Y}_{\mathcal{B}} \mid \mathbf{Y}_{\mathcal{C}}
$$

for disjoint sets $\mathcal{A}, \mathcal{B}$, and $\mathcal{C}$, with $\mathcal{A}$ and $\mathcal{B}$ non-empty, where the set $\mathcal{C}$ separates $\mathcal{A}$ and $\mathcal{B}$ i.e., on removing the nodes in $\mathcal{C}$ from the graph, nodes in $\mathcal{A}$ are no longer connected to the nodes in $\mathcal{B}$.

Thus, in (6), the local-Markov property states that the conditional distribution at a node in the DG given the observations at its neighbors is independent of the rest of the network. By the global-Markov property in (7), all the connected components of a dependency graph are independent. As an illustration, in Fig. 2

$$
\left[\begin{array}{ccccccccc}
\times & & \times & & & & & \\
& \times & \times & & & & & \\
\times & \times & \times & & \times & & & \\
& & & \times & \times & & & \\
& & \times & \times & \times & \times & & \\
& & & & \times & \times & & \\
& & & & & & \times & \times \\
& & & & & & \times & \times
\end{array}\right]
$$

(b) $x$ : Non-zero elements of potential matrix. we have $Y_{6} \perp Y_{7}$ given the rest of network, $Y_{1} \perp Y_{2} \mid Y_{3}$, and so on.

\section{ACYCLIC DEPENDENCY GRAPH}

A special case of the dependency graph is an acyclic or a cycle-free graph. Here, the neighbors of a node are not themselves neighbors. The joint distribution is somewhat easier to evaluate in this case. We note that an acyclic graph with at least one edge, always has a leaf i.e., it has a node with degree 1 and has utmost $n-1$ edges in a $n$-node graph.

The covariance matrix $\boldsymbol{\Sigma}$ of a GMRF satisfies some special properties. For instance, consider the cross covariance between the neighbors of a node, i.e., nodes that are two hops away in an acyclic DG. By the global-Markov property we have ${ }^{2}$, assuming $\Sigma(i, i)>0$, for $i \in \mathcal{V}, \operatorname{Deg}(i) \geq 2, j, k \in \mathcal{N}(i), j \neq k$

$$
\Sigma(j, k)=\frac{\Sigma(i, j) \Sigma(i, k)}{\Sigma(i, i)} .
$$

For example, in Fig. 2

$$
\Sigma(1,2)=\frac{\Sigma(1,3) \Sigma(2,3)}{\Sigma(3,3)} .
$$

We can similarly find an expression for the covariance between any two nodes of the GMRF. Thus, the covariance matrix of a GMRF with acyclic dependency can be expressed solely in terms of the auto covariance of the nodes and the cross covariance between the neighbors of the dependency graph.

\section{A. Potential Matrix}

The inverse of the covariance matrix of a non-degenerate GMRF ( i.e., with a positive-definite covariance matrix) is known as the potential matrix or the precision matrix or the information matrix. The non-zero elements of the potential matrix $\mathbf{A}$ are in one to one correspondence with the edges of its graph $\mathcal{G}(\mathcal{V}, \mathcal{E})[8$, Theorem 2.2] in the sense that

$$
i \nsim j \Longleftrightarrow A(i, j)=0, \forall i, j \in \mathcal{V}, i \neq j
$$

and is illustrated in Fig. 2.

This simple correspondence between the conditional independence of the GMRF and the zero structure of its potential matrix is not evident in the covariance matrix, which is generally a completely dense matrix. Therefore, it is easier to evaluate the

\footnotetext{
${ }^{2}$ For $X, Y$ jointly zero mean Gaussian, $\mathbb{E}(\mathbf{X} \mid \mathbf{y})=\Sigma_{x y} \Sigma_{y y}^{-1} \mathbf{y}$.
} 
joint distribution of the GMRF through the potential matrix. In practice, however, estimates of the covariance matrix are easier to obtain through the empirical observations. Therefore, it is desirable to have the joint distribution in terms of coefficients of the covariance matrix. Thus, an explicit expression between the coefficients of the covariance and the potential matrix is needed. We provide such an expression and also obtain the determinant of the potential matrix in the theorem below.

Theorem 1 (Elements and Determinant of Potential Matrix): The elements of the potential matrix $\mathbf{A}:=\boldsymbol{\Sigma}^{-1}$, for a positive-definite covariance matrix $\boldsymbol{\Sigma}$ and acyclic dependency graph $\mathcal{G}(\mathcal{V}, \mathcal{E})$, are

$$
\begin{aligned}
& A(i, i)=\frac{1}{\Sigma(i, i)}\left(1+\sum_{j \in \mathcal{N}(i)} \frac{\Sigma(i, j)^{2}}{\Sigma(i, i) \Sigma(j, j)-\Sigma(i, j)^{2}}\right) \\
& A(i, j)= \begin{cases}\frac{-\Sigma(i, j)}{\Sigma(i, i) \Sigma(j, j)-\Sigma(i, j)^{2}}, & \text { if } i \sim j \\
0, & \text { o.w. }\end{cases}
\end{aligned}
$$

The determinant of the potential matrix of $\mathbf{A}$ is given by

$$
|\mathbf{A}|=\frac{1}{|\boldsymbol{\Sigma}|}=\frac{\prod_{\substack{i \in \mathcal{V} \\ \Sigma}(i, i)^{\operatorname{Deg}(i)}-1}\left[\Sigma(i, i) \Sigma(j, j)-\Sigma(i, j)^{2}\right]}{\prod_{i<j}[}
$$

Proof: The proof is based on acyclicity of dependency graph. See Appendix A.

\section{Hypothesis-Testing Problem}

Let $\mathcal{V}=\{1, \ldots, n\}$ be a set of $n$ nodes on the plane and let $\mathbf{Y}_{n}$ be the random vector of observation samples $Y_{i}, i \in \mathcal{V}$

$$
\mathbf{Y}_{n}:=\left[Y_{1}, \ldots, Y_{n}\right]^{T}
$$

The hypothesis-testing problem is as follows (also see Fig. 1)

$$
\mathcal{H}_{0}: \mathbf{Y}_{n} \sim \mathcal{N}\left(\mathbf{0}, \sigma_{0}^{2} \mathbf{I}\right) \quad \text { versus } \quad \mathcal{H}_{1}: \mathbf{Y}_{n} \sim \mathcal{N}\left(\mathbf{0}, \boldsymbol{\Sigma}_{1, \mathcal{V}}\right)
$$

where $\Sigma_{1, \mathcal{V}}$ is a positive-definite covariance matrix under the alternative hypothesis and is dependent on the configuration of nodes in $\mathcal{V}$ and $\sigma_{0}^{2}>0$ is the uniform variance under the null hypothesis.

The optimal decision-rule under both NP and Bayesian formulations is a threshold test based on the log-likelihood ratio (LLR). Let $p\left[\mathbf{Y}_{n} \mid \mathcal{V} ; \mathcal{H}_{j}\right]$ be the conditional PDF of the observations given the point set $\mathcal{V}$ under hypothesis $j$. The LLR given by

$$
\begin{aligned}
\operatorname{LLR}\left(\mathbf{Y}_{n}, \mathcal{V}\right): & =\log \frac{p\left[\mathbf{Y}_{n}, \mathcal{V} ; \mathcal{H}_{0}\right]}{p\left[\mathbf{Y}_{n}, \mathcal{V} ; \mathcal{H}_{1}\right]}=\log \frac{p\left[\mathbf{Y}_{n} ; \mathcal{H}_{0}\right]}{p\left[\mathbf{Y}_{n} \mid \mathcal{V} ; \mathcal{H}_{1}\right]} \\
& =\frac{1}{2}\left(\log \frac{\left|\boldsymbol{\Sigma}_{1, \mathcal{V}}\right|}{\left|\sigma_{0}^{2} \mathbf{I}\right|}+\mathbf{Y}_{n}^{T}\left[\boldsymbol{\Sigma}_{1, \mathcal{V}}^{-1}-\left(\sigma_{0}^{2} \mathbf{I}\right)^{-1}\right] \mathbf{Y}_{n}\right)
\end{aligned}
$$

where in (15), we have used the fact that the sensor observations are independent of $\mathcal{V}$ under $\mathcal{H}_{0}$.

\section{A. Covariance Matrix of GMRF}

We make additional assumption on the structure of the covariance matrix $\boldsymbol{\Sigma}_{1, \mathcal{V}}$ of the GMRF under $\mathcal{H}_{1}$ viz., that the nodes have the same measurement variance for any node configuration $\mathcal{V}$, i.e.,

$$
\Sigma_{1, \mathcal{V}}(i, i):=\sigma_{1}^{2}>0, \quad i=1, \ldots, n .
$$

We denote the ratio between the variances under the alternative and the null hypothesis at each node by

$$
K:=\frac{\sigma_{1}^{2}}{\sigma_{0}^{2}} \text {. }
$$

We also assume that under $\mathcal{H}_{1}$, the amount of correlation between the neighbors $i, j$ of the dependency graph is specified by an arbitrary function $g$, which has the Euclidean edge length $R_{i j}$ as its argument. From (16), we have

$$
g\left(R_{i j}\right):=\frac{\Sigma_{1, \mathcal{V}}(i, j)}{\sigma_{1}^{2}}<1, \quad \forall(i, j) \in \mathcal{E} .
$$

The correlation function $g$ is required to satisfy some regularity conditions, which will be stated in Lemma 2. In general, $g$ is a monotonically nonincreasing function of the edge length, since amount of correlation usually decays as nodes become farther apart. Moreover, $g(0)=M<1$, or the so-called nugget effect, according to geostatistics literature [35], [36]. It has been observed in mining applications, where the microscale variation is assumed to be caused by the existence of small nuggets of the enriched ore. Many other ecological phenomena such as soil bacteria population [37], aquatic population [38] etc. also exhibit this behavior. Note that the presence of nugget effect has the same effect on correlation as imposing an exclusion region on how near two nodes can be placed. However, for such an exclusion constraint to hold, we need more complicated node placement distributions than the uniform or Poisson assumption. Although such distributions can be handled in principle, they are not analytically tractable.

Some examples of the correlation function are

$$
g(R)=M \mathrm{e}^{-a R}, g(R)=\frac{M}{1+R^{a}}, \quad a \geq 0,0 \leq M<1 .
$$

Sufficient conditions will be later imposed on the limiting correlation $M$ to ensure positive definiteness.

\section{B. Nearest-Neighbor Graph}

We assume the dependency graph to be the nearest-neighbor graph. The nearest-neighbor function of a node $i \in \mathcal{V}$, is defined as

$$
\mathrm{nn}(i):=\arg \min _{j \in \mathcal{V}, j \neq i} \operatorname{dist}(i, j)
$$

where $\operatorname{dist}(\cdot, \cdot)$ is the Euclidean distance. The inter-point distances are unique with probability 1 , for uniform and Poisson point sets under consideration here. Therefore, $\operatorname{nn}(i)$ is a well-defined function almost surely. The nearest-neighbor (undirected) graph $\mathcal{G}(\mathcal{V}, \mathcal{E})$ is given by

$$
(i, j) \in \mathcal{E} \Longleftrightarrow i=\mathrm{nn}(j) \text { or } j=\mathrm{nn}(i) \text {. }
$$




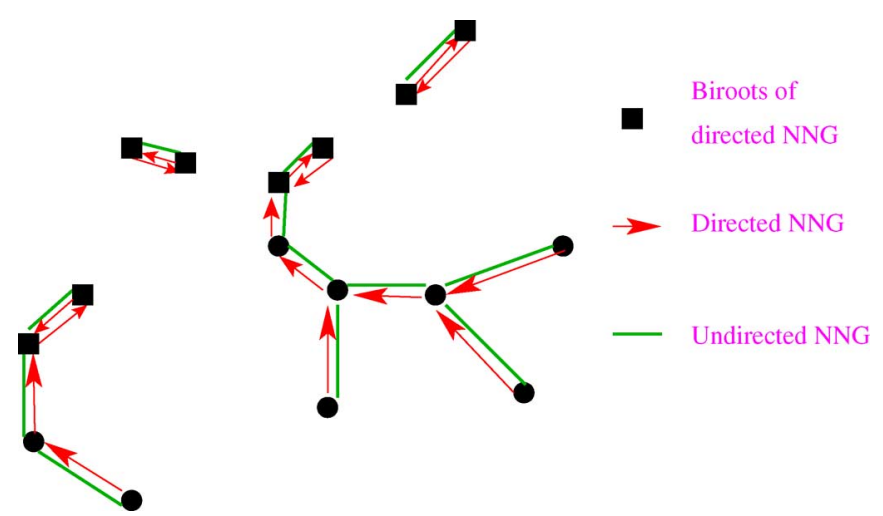

Fig. 3. Directed and undirected versions of NNG. [No. of undirected edges $=$ No. of directed edges $-0.5 \times$ No. of biroots.].

NNG has a number of important properties. It is acyclic with a maximum ${ }^{3}$ node degree of 5 almost surely [40].

In Section VI-C, it turns out that we need to analyze the directed NNG, in order to obtain the final form of the error exponent. We now mention some of its special properties. The directed $\mathrm{NNG} \mathcal{G}^{\prime}\left(\mathcal{V}, \mathcal{E}^{\prime}\right)$ is defined by

$$
\mathcal{E}^{\prime}=\{<i, \operatorname{nn}(i)>, i \in \mathcal{V}\}
$$

For a directed NNG with at least two nodes, each connected component contains exactly one two-cycle. This is known as the biroot of the component [40]. See Fig. 3. Also note, the directed NNG counts the edges from these biroots twice, while the undirected version counts only once.

\section{Expression for Log-Likelihood Ratio}

Since the NNG is acyclic, (11)-(12) are valid. We incorporate additional assumptions (16)-(18) in the theorem to obtain the LLR for detection.

We now impose additional constraints to ensure that the potential matrix (and hence, also the covariance matrix) is positive definite through diagonal dominance. It can be shown that a sufficient condition for this is $M<\frac{1}{\mathrm{Deg}_{\max -1}}=\frac{1}{4}$, since the NNG has a maximum degree of 5 almost surely. This is only a sufficient condition, and simulations show that larger values of $M$ are possible.

Theorem 2 (Log-Likelihood Ratio): Under the assumptions (16)-(18), the log-likelihood ratio in (15) for the hypothesis-testing problem in (14), given an arbitrary point set $\mathcal{V}=\{1, \ldots, n\}$, is

$$
\begin{aligned}
\operatorname{LLR}\left(\mathbf{Y}_{n}, \mathcal{V}\right)= & n \log \frac{\sigma_{1}}{\sigma_{0}}+\frac{1}{2}\left[\sum_{i \in \mathcal{V}}\left(\frac{1}{\sigma_{1}^{2}}-\frac{1}{\sigma_{0}^{2}}\right) Y_{i}^{2}\right. \\
& +\sum_{\substack{(i, j) \in \mathcal{E} \\
i<j}}\left\{\log \left[1-g^{2}\left(R_{i j}\right)\right]\right. \\
& +\frac{g^{2}\left(R_{i j}\right)}{1-g^{2}\left(R_{i j}\right)} \frac{Y_{i}^{2}+Y_{j}^{2}}{\sigma_{1}^{2}} \\
& \left.\left.-\frac{2 g\left(R_{i j}\right)}{1-g^{2}\left(R_{i j}\right)} \frac{Y_{i} Y_{j}}{\sigma_{1}^{2}}\right\}\right]
\end{aligned}
$$

${ }^{3}$ The node degree is finite for NNG in any dimension and is called the kissing number [39]. where $R_{i j}$ is the Euclidean edge length of $(i, j) \in \mathcal{E}$, that depends on the configuration of $\mathcal{V}$. The condition $i<j$ ensures that every edge is counted only once.

Theorem 2 gives a closed-form expression for the LLR, in terms of the edges of the nearest-neighbor dependency graph of the GMRF. Note in (22), the cross-terms are only between the neighbors of the dependency graph, which can be exploited to yield explicit data-fusion and routing schemes [41].

\section{NEYMAN-PEARSON ERROR EXPONENT}

The spectrum of the log-likelihood ratio is defined as the distribution of the normalized LLR evaluated under the null hypothesis. In [25, Theorem 1], it is proven that for Neyman-Pearson detection under a fixed type-I error bound, ${ }^{4}$ the LLR spectrum can fully characterize the type-II error exponent of the hypothesis-testing system and is independent of the type-I bound.

A special case of this result is when the LLR spectrum converges almost surely (a.s.) to a constant $D$

$$
\frac{1}{n} \operatorname{LLR}\left(\mathbf{Y}_{n}, \mathcal{V}\right)=\frac{1}{n} \log \frac{p\left[\mathbf{Y}_{n} ; \mathcal{H}_{0}\right]}{p\left[\mathbf{Y}_{n} \mid \mathcal{V} ; \mathcal{H}_{1}\right]} \stackrel{\text { a.s. }}{\rightarrow} D, \quad \text { under } \mathcal{H}_{0} .
$$

In this case, the NP type-II error exponent is given by the above constant $D$. In other words, the error exponent $D$ of NP detection in (1) is

$$
D:=\lim _{n \rightarrow \infty} \frac{1}{n} \log \frac{p\left[\mathbf{Y}_{n} ; \mathcal{H}_{0}\right]}{p\left[\mathbf{Y}_{n} \mid \mathcal{V} ; \mathcal{H}_{1}\right]}, \quad \text { under } \mathcal{H}_{0}
$$

where lim denotes the almost-sure limit, assuming it exists. Note that when $\mathbf{Y}_{n}$ are i.i.d. conditioned under either $\mathcal{H}_{0}$ or $\mathcal{H}_{1}$, the result reduces to the Stein's lemma [43, Theorem 12.8.1] and the limit in (23) to the Kullback-Leibler distance.

\section{A. Random Point Sets}

It is intractable to evaluate the error exponent $D$ in (23) for an arbitrary point set. Therefore, we assume that the nodes are placed randomly, according to a point process defined on expanding regions. We consider two related point processes : the Poisson process and the binomial point process on a large region, which we define below.

Definition 2 (Poisson and Binomial Processes [44]): Let $\left(\mathcal{B}_{n}\right)_{n \geq 1}$ denote a sequence of squares or circles 5 of area $\frac{n}{\lambda}$, centered at the origin, for any $\lambda>0$. A binomial point process on $\mathcal{B}_{n}$, denoted by $\mathcal{U}_{n, \lambda}$, consists of $n$ points distributed i.i.d. uniformly on $\mathcal{B}_{n}$. A homogeneous Poisson process of intensity $\lambda$ on $\mathcal{B}_{n}$, denoted by $\mathcal{P}_{n, \lambda}$, satisfies the following properties:

1) for any set $\mathcal{A} \subset \mathcal{B}_{n}$ with area $A$, the number of points in $\mathcal{A}$ is Poisson distributed with mean $\lambda A$;

2) for any $n \in \mathbb{N}$ and $\mathcal{A} \subset \mathcal{B}_{n}$ with area $A>0$, conditioned on $n$ number of points in $\mathcal{A}$, the point process on $\mathcal{A}$ is a binomial process.

We are interested in evaluating the error exponent under both the binomial or Poisson point processes, when the mean number

\footnotetext{
${ }^{4}$ The generalization to an exponential type-I error bound [25], [42] is not
} tractable since a closed-form cumulative distribution of the LLR is needed.

${ }^{5}$ The results hold for regular Borel sets under some conditions [4, p. 1007]. 


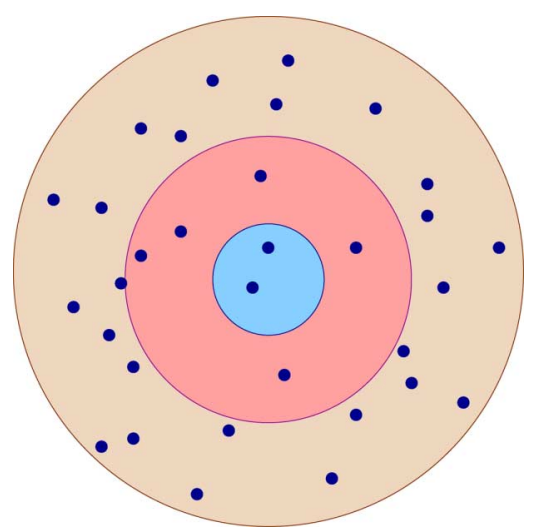

Fig. 4. Illustration of point process $\mathcal{U}_{n, \lambda}$ or $\mathcal{P}_{n, \lambda}: n$ nodes distributed i.i.d. uniform or Poisson in regular Borel regions (such as squares or circles) of area $\frac{n}{\lambda}$ with constant density $\lambda$. For error exponent, we consider $n \rightarrow \infty$.

of nodes goes to infinity, with fixed node density, i.e., $n \rightarrow \infty$ with $\lambda$ fixed.

\section{Closed-Form ERROR EXPONENT}

\section{A. Error Exponent as a Graph Functional}

In order to derive the error exponent, we cast the error exponent as the limit of sum of node and edge functionals of the dependency graph of a marked point set in the lemma below. This formulation is required in order to apply the law of large numbers for graph functionals.

Lemma 1 ( $D$ as a Graph Functional): Given the marked point set $\mathcal{V}$ drawn from the binomial process $\mathcal{U}_{n, \lambda}$ or the Poisson process $\mathcal{P}_{n, \lambda}$, with marking variable $Y_{i} \stackrel{\text { i.i.d. }}{\sim} \mathcal{N}\left(0, \sigma_{0}^{2}\right)$, the error exponent $D$ in (23) is given by the limit of sum of edge and node functionals of the nearest-neighbor graph as

$$
\begin{aligned}
D= & \log \frac{\sigma_{1}}{\sigma_{0}}+\lim _{n \rightarrow \infty} \frac{1}{2 n}\left[\sum_{i \in \mathcal{V}}\left(\frac{1}{\sigma_{1}^{2}}-\frac{1}{\sigma_{0}^{2}}\right) Y_{i}^{2}\right. \\
& +\sum_{\substack{(i, j) \in \mathcal{E} \\
i<j}}\left\{\log \left[1-g^{2}\left(R_{i j}\right)\right]+\frac{g^{2}\left(R_{i j}\right)}{1-g^{2}\left(R_{i j}\right)} \frac{Y_{i}^{2}+Y_{j}^{2}}{\sigma_{1}^{2}}\right. \\
& \left.\left.-\frac{2 g\left(R_{i j}\right)}{1-g^{2}\left(R_{i j}\right)} \frac{Y_{i} Y_{j}}{\sigma_{1}^{2}}\right\}\right], \quad Y_{i} \stackrel{\text { i.i.d. }}{\sim} \mathcal{N}\left(0, \sigma_{0}^{2}\right)
\end{aligned}
$$

where $R_{i j}$ is the (random) Euclidean edge length of $(i, j) \in$ $\mathcal{E}$, that depends on the underlying point process. The condition $i<j$ ensures that every edge is counted only once.

Proof: Substitute (22) in (23).

In the lemma above, the point set forming the graph is drawn from a marked binomial or Poisson point process, with the marking variable $Y_{i} \stackrel{\text { i.i.d. }}{\sim} \mathcal{N}\left(0, \sigma_{0}^{2}\right)$. This is because evaluating the error exponent (23) under $\mathcal{H}_{0}$ implies that the sensor observations $Y_{i}$ are i.i.d. and independent of the locations of the nodes, and therefore can be viewed as a marking process.

\section{B. Law of Large Numbers for Graph Functionals}

The law of large numbers for functionals on graphs enables us to evaluate the $\operatorname{limit}^{6}$ in (24). This law applies to graphs which are random in the sense that the vertex set is a marked random point set. LLN on graphs is based on the so-called objective method. Steele [45] coined this term for a philosophy whereby, loosely speaking, one describes the limiting behavior of functionals on finite point sets of binomial process in terms of related functionals defined on infinite Poisson point sets. Also see Fig. 5. Penrose and Yukich [4], [33], and [34] introduce a concept of stabilizing functionals and use the objective method to establish a strong law of large numbers for graph functionals [33, p. 287]. In order to apply this law, some conditions need to be satisfied in terms of bounded moments. In the lemma below, we place these conditions on the correlation function.

Lemma 2 (Conditions for $L L N$ ): The graph functional in (24) satisfies the conditions for law of large numbers for graph functionals derived in [33, p. 287], when the correlation function $g$ is monotonically nonincreasing with the edge-lengths, $g(\infty)=0$, and $g(0)=M<1$. Hence, the graph functional in (24) converges almost surely to a constant.

Proof: See Appendix B.

Theorem 3 (LLN): Under the conditions stated in Lemma 2, for nodes placed according to $\mathcal{U}_{n, \lambda}$ or $\mathcal{P}_{n, \lambda}$, with node density $\lambda$ and region area $\frac{n}{\lambda}$, from the law of large numbers for graph functionals, the expression for the error exponent $D$ in (24) for Neyman-Pearson detection of the GMRF defined by the NNG is given by

$$
D=\frac{1}{2}\left[\frac{1}{2} \mathbb{E} \sum_{\substack{\mathbf{X}: \mathbf{X} \in \mathcal{P}_{\lambda},(\mathbf{0}, \mathbf{X}) \in \operatorname{NNG}(\mathbf{X} \cup \mathbf{0})}} f\left(g\left(R_{\mathbf{0}, \mathbf{X}}\right)\right)+\log K+\frac{1}{K}-1\right]
$$

where

$$
f(x):=\log \left[1-x^{2}\right]+\frac{2 x^{2}}{K\left[1-x^{2}\right]}
$$

$K$ is the ratio of variances defined in (17), and $R_{\mathbf{0}, \mathbf{X}}$ are the (random) lengths of edge $(\mathbf{X}, \mathbf{0})$ incident on the origin in a NNG, when the nodes are distributed according to homogeneous Poisson process $\mathcal{P}_{\lambda}$, of intensity $\lambda$.

Proof: Apply LLN to (24). See Appendix C.

In the theorem above, the law of large numbers yields the same limit ${ }^{7}$ under the Poisson or the binomial process. Thus, we provide a single expression for the error exponent under both the processes. Also, the above theorem provides the error exponent in terms of the expectation of a graph functional around the origin, with the points drawn from an infinite Poisson process. Thus, the functional is reduced to a localized effect around the origin. This is an instance of the broad concept of stabilization which states that the local behavior of the graph in a bounded

\footnotetext{
${ }^{6}$ Nature of convergence is convergence of means and complete convergence (c.m.c.c) and implies almost-sure convergence.

${ }^{7}$ In general, the limit is not the same for Poisson and binomial processes. For a different problem, we show that the error exponents are affected by a random sample size [46].
} 


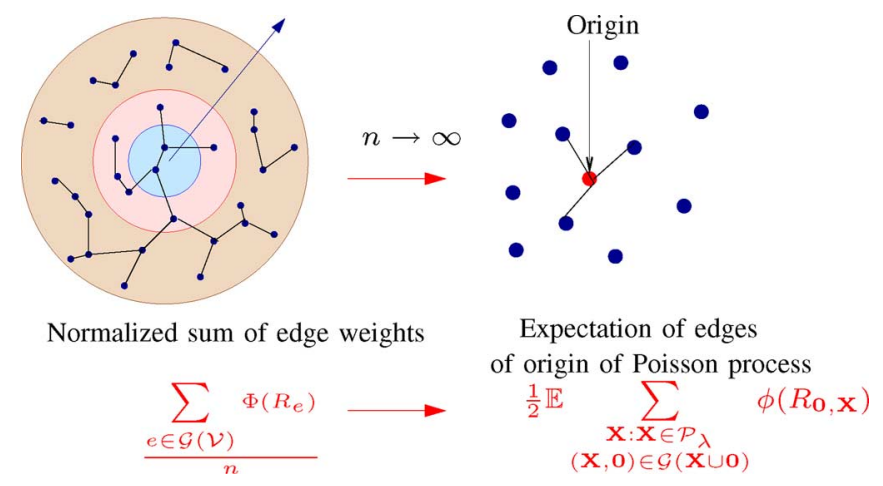

Fig. 5. Pictorial representation of LLN for graph functionals of uniform or Poisson point sets.

region is unaffected by points beyond a finite (but random) distance from that region. NNG is one such stabilizing graph with translation and scale-invariance [4, Lemma 6.1].

\section{Explicit Form for Nearest-Neighbor Graph}

The evaluation of the expectation of the edge functional in (25) is complicated and needs further simplification. In order to obtain the final form of the exponent, we exploit some special properties of the NNG. It turns out that the expectation term is easier to evaluate for the directed nearest-neighbor graph rather than the undirected version. We therefore split the sum of edge functionals in (24), using the fact that the directed NNG counts the weights from biroots or mutual neighbors twice, while the undirected version counts only once. See Fig. 3. We therefore split the sum of the edge functionals of the undirected NNG as

$$
\begin{aligned}
\sum_{e \in \mathrm{NNG}(\mathcal{V})} f\left(g\left(R_{e}\right)\right)= & \sum_{e \in \mathrm{DNNG}(\mathcal{V})} f\left(g\left(R_{e}\right)\right) \\
& -\frac{1}{2} \sum_{e \in \mathrm{MNNG}(\mathcal{V})} f\left(g\left(R_{e}\right)\right)
\end{aligned}
$$

where $\mathrm{NNG}(\mathcal{V}), \mathrm{DNNG}$ and MNNG $\subset \mathrm{DNNG}$ are the undirected NNG, the directed NNG, and edges between the biroots or the mutual neighbors of the directed NNG, respectively. Now, we evaluate the expectation for the two terms separately, since expectation is linear. A similar approach is employed in [47].

We now provide an expression for the limit of the edge functional based on the distribution of distances of the directed NNG, which are related to hitting or vacancy probabilities of the spatial point process, which are typically exponential or gamma distributed, similar to their one-dimensional counterparts [48].

Lemma 3 (Expectation of Edge Functional): The expectation term of the edge functional in (25) is given by

$\frac{1}{2} \mathbb{E} \quad \sum_{\mathbf{X}: \mathbf{X} \in \mathcal{P}_{\lambda},} f\left(g\left(R_{\mathbf{0}, \mathbf{X}}\right)\right)=\mathbb{E} f\left(g\left(Z_{1}\right)\right)-\frac{\pi}{2 \omega} \mathbb{E} f\left(g\left(Z_{2}\right)\right)$, $(\mathbf{0}, \mathbf{X}) \in \mathrm{NNG}(\mathbf{X} \cup \mathbf{0})$

where $Z_{1}$ and $Z_{2}$ are Rayleigh distributed with variances $(2 \pi \lambda)^{-1}$ and $(2 \omega \lambda)^{-1}$, and $\omega$ is given by

$$
\omega=\frac{4 \pi}{3}+\frac{\sqrt{3}}{2} \approx 5.06
$$

and is the area of the union of two unit- radii circles with centers unit distant apart.

Proof: See Appendix D.

In the theorem below, we combine Lemmas 2,3 , and Theorem 3 to obtain the final form of the error exponent.

Theorem 4 (Expression for D): Under the assumptions (16)-(18) and conditions stated in Lemma 2, for a GMRF with NNG dependency and correlation function $g$ and nodes drawn from the binomial or the Poisson process with node density $\lambda$ and region area $\frac{n}{\lambda}$, the error exponent $D$ for Neyman-Pearson detection is

$$
\begin{array}{r}
D_{g}(K, M, \lambda)=\frac{1}{2}\left[\mathbb{E} f\left(g\left(Z_{1}\right), K\right)-\right. \\
\frac{\pi}{2 \omega} \mathbb{E} f\left(g\left(Z_{2}\right), K\right) \\
\left.+\log K+\frac{1}{K}-1\right]
\end{array}
$$

where

$$
f(x, K):=\log \left[1-x^{2}\right]+\frac{2 x^{2}}{K\left[1-x^{2}\right]} .
$$

$Z_{1}$ and $Z_{2}$ are Rayleigh distributed with second moments $(2 \pi \lambda)^{-1}$ and $(2 \omega \lambda)^{-1}$.

The above theorem holds for any general correlation function. In (30), except for the first two $f$-terms which capture the correlation structure of the GMRF, the remaining terms represent the detection error exponent for two i.i.d. Gaussian processes. In the corollary below, we specialize (30) to the case of constant correlation. In this case, the two $f$-terms reduce to a single term.

Corollary 1 (Constant Correlation): For constant values of the correlation, the error exponent $D$ is independent of the node density $\lambda$ and

1) for constant positive correlation or $g\left(R_{e}\right) \equiv M<1$, $\forall e \in$ $\mathcal{E}$, we have

$$
D(K, M)=\frac{1}{2}\left[\log K+\frac{1}{K}-1+\left(1-\frac{\pi}{2 \omega}\right) f(M, K)\right],
$$

where $f$ and $\omega$ are given by (26) and (29).

2) for the independent case or $g\left(R_{e}\right) \equiv 0, \forall e \in \mathcal{E}$, we have

$$
D(K, 0)=\frac{1}{2}\left[\log K+\frac{1}{K}-1\right] .
$$

In the above corollary, we verify that (32) reduces to (33), on substituting $M=0$. In (32), the effect of correlation can be easily analyzed through the sign of the function $f(M, K)$. Also,

$$
f(M, K) \begin{cases}<0, & \text { for } K>\frac{2}{1-M^{2}} \\ >0, & \text { for } K<2 .\end{cases}
$$

Therefore, at large variance-ratios, the presence of correlation hurts the asymptotic performance, when compared with the independent case. But the situation is reversed at low values of the variance ratio and the presence of correlation helps in detection performance. In the next section, we will draw similar conclusions when the correlation function is the exponential function through numerical evaluations. 


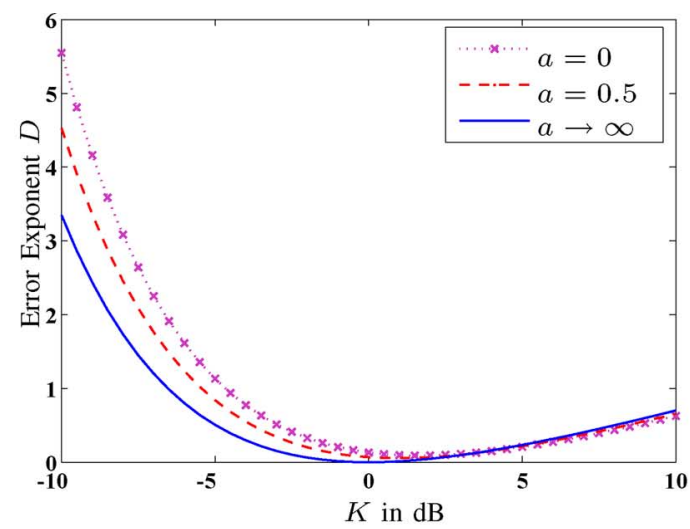

(a) Different values of correlation coefficient $a$, nugget $M=0.5$.

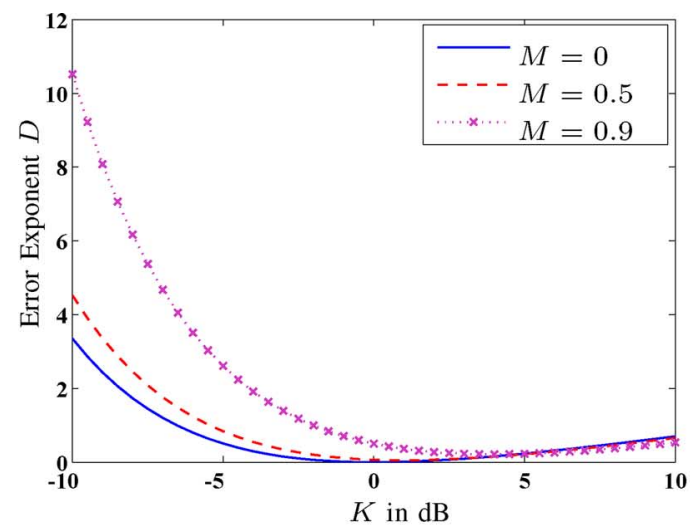

(b) Different values of nugget $M$, correlation coefficient $a=0.5$.

Fig. 6. Error exponent $D$ versus ratio of variances $K$, node density $\lambda=1$. See (32)-(35).

\section{Numerical Results}

In this section, we focus on a specific correlation function namely the exponential-correlation function,

$$
g(R)=M \mathrm{e}^{-a R}, \quad a>0,0<M<1 .
$$

Using Theorem 4, we numerically evaluate $D$ through MonteCarlo runs. In (30), the error exponent is an implicit function of the correlation coefficient $a$, through the correlation function $g$. For fixed values of $K$ and $M$, we have

$$
D(K, M, \lambda, a)=D\left(K, M, 1, \frac{a}{\sqrt{\lambda}}\right)
$$

which we obtain by changing the integration variable in the expectation term in (30). Therefore, in terms of the error exponent, increasing the node density $\lambda$ is equivalent to a lower correlation coefficient at unit density. Here, we plot only the effects of correlation coefficient $a$ and nugget $M$ on $D$.

In Fig. 6(a), we plot the error exponent at $\lambda=1$ and $M=0.5$, for different values of correlation coefficient $a$. Note, the cases $a=0$ and $a \rightarrow \infty$ correspond to (32) and (33). We notice that a more correlated GMRF or the one with smaller $a$, has a higher exponent at low value of $K$, whereas the situation is reversed at high $K$. Equivalently, increasing the node density $\lambda$ improves the exponent at low value of $K$, but not at high $K$. Also, when the variance ratio $K$ is large enough, $D$ appears to increase linearly with $K$ (in decibels), and the correlation coefficient $a$ and nugget $M$ appear to have little effect, as expected from Theorem 4. In Fig. 6(b), we plot the exponent at constant correlation coefficient $a=0.5$ for different values of the nugget $M$. Also note, $M=0$ reduces to the independent case. We notice a similar behavior as the correlation coefficient. A higher value of $M$ results in a higher exponent at low $K$, but not at high $K$.

\section{CONCLUSION}

In general, finding the closed-form detection error exponent is not tractable. The graphical structure of the Markov random field allows us to exploit existing results in spatial probability literature. We employed the law of large numbers for graph functionals to derive the detection error exponent for a GaussMarkov random field with nearest-neighbor dependency graph. We then investigated the influence of model parameters such as the variance ratio and the correlation function on the error exponent.

In this paper, we have assumed identical variance at every sensor. However, a spatially varying SNR model can be incorporated into our results. We have focused on the GMRF defined by the acyclic dependency graph and derived the exponent for the nearest-neighbor graph. This is a simplifying assumption. Although, the law of large numbers is valid for a number of proximity graphs, which have edges between "nearby" points, the actual evaluation of the LLR and the exponent are intractable for most of these graphs. We have not considered correlation under null hypothesis for which one requires a LLN with correlated marks. We have also not considered the case when the signal field is not directly observable, resulting in a hidden GMRF. The sparse structure of the potential matrix is no longer valid under such a scenario. However, note, GMRF with small neighborhood has been demonstrated to approximate the hidden GMRF [49] as well as Gaussian field with long correlation lengths [50], reasonably well.

The error exponent can be employed as a performance measure for network design. In [51], we utilize the closed form derived in this paper to obtain an optimal node density that maximizes the exponent subject to a routing energy constraint. We have also proposed minimum energy data fusion and routing schemes that exploit the correlation structure of Markov random field in a related publication [41]. We further investigate tradeoffs between the routing energy and the resulting error exponent in [52].

\section{APPENDIX A \\ PROOF OF THEOREM 1}

Using the expression $\mathbf{A} \boldsymbol{\Sigma}=\mathbf{I}$, we have the following identities:

$$
\begin{aligned}
& A(i, i)+\sum_{j \in \mathcal{N}(i)} A(i, j) \frac{\Sigma(i, j)}{\Sigma(i, i)}=\frac{1}{\Sigma(i, i)} \\
& A(i, i)+A(i, j) \frac{\Sigma(j, j)}{\Sigma(i, j)}+\sum_{\substack{k \in \mathcal{N}(i) \\
k \neq j}} A(i, k) \frac{\Sigma(j, k)}{\Sigma(i, j)}=0 \\
& \forall j \in \mathcal{N}(i)
\end{aligned}
$$

where (37) is obtained by the sum-product of $i$ th row and $i$ th column of $\mathbf{A}$ and $\boldsymbol{\Sigma}$. Similarly, (38) is obtained by sum-product 
of $i$ th row of $\mathbf{A}$ and $j$ th column of $\boldsymbol{\Sigma}$ and dividing by $\Sigma(i, j)$. In (38), by acyclicity for $k \in \mathcal{N}(i)$ and $k \neq j$, we have $j \nsim k$. From (8), we have

$$
\frac{\Sigma(j, k)}{\Sigma(i, j)}=\frac{\Sigma(i, k)}{\Sigma(i, i)}, \quad \forall j, k \in \mathcal{N}(i), k \neq j .
$$

Subtracting (38) from (37), only the terms with $A(i, j)$ survive and hence, we obtain $A(i, j)$. Substituting all the $A(i, j)$ 's in (37), we obtain $A(i, i)$. Hence, all the coefficients of potential matrix $\mathbf{A}$ are given by (11).

Let $\left|\mathbf{A}^{(n)}\right|$ be the determinant of the potential matrix of $n$ nodes. Assume $n>1$, since we have $\left|\mathbf{A}^{(1)}\right|=\Sigma(1,1)^{-1}$. The determinant of the potential matrix is the product of determinants of the connected components. We therefore consider only one component $\mathcal{G}^{\prime}\left(\mathcal{V}^{\prime}, \mathcal{E}^{\prime}\right) \subseteq \mathcal{G}$. Assume $\mathcal{G}^{\prime}$ has at least one edge, otherwise we have for diagonal matrix $\left|\mathbf{A}^{(n)}\right|=\prod_{i \in \mathcal{V}^{\prime}} \Sigma(i, i)^{-1}$. Since $\mathcal{G}^{\prime}$ is acyclic, it has a leaf, i.e., there is some vertex $a$ with degree 1 . Let $b$ be its only neighbor. We assume the vertices have been ordered $\mathcal{V}^{\prime}=\left\{V_{1}, \ldots, V_{n}\right\}$ so that $V_{n-1}=b, V_{n}=a$. Then $\mathbf{A}^{(n)}$ has the following form:

$$
\mathbf{A}^{(n)}=\left[\begin{array}{ccccc}
\cdot & \cdots & \cdot & \cdot & 0 \\
\vdots & \vdots & \vdots & \vdots & \vdots \\
\cdot & \cdots & \cdot & \cdot & 0 \\
\cdot & \cdots & \cdot & A(n-1, n-1) & A(n-1, n) \\
0 & \cdots & 0 & A(n, n-1) & A(n, n)
\end{array}\right],
$$

where we have from (11)

$$
\begin{aligned}
A(n, n)= & \frac{\Sigma(n-1, n-1)}{\left[\sum(n, n) \Sigma(n-1, n-1)-\Sigma(n, n-1)^{2}\right]} \\
A(n-1, n)= & \frac{-\Sigma(n, n-1)}{\left[\Sigma(n, n) \Sigma(n-1, n-1)-\Sigma(n, n-1)^{2}\right]} \\
A(n-1, n-1)= & \frac{1}{\sum(n-1, n-1)} \\
& -A(n-1, n) \frac{\Sigma(n, n-1)}{\Sigma(n-1, n-1)}+C
\end{aligned}
$$

where $C$ represents contributions from nodes in $\mathcal{V}^{\prime} \backslash V_{n}$ i.e., with node $V_{n}$ removed, and having an edge with $V_{n-1}$. Multiplying the $n$th column by

$$
\frac{A(n, n-1)}{A(n, n)}=\frac{-\Sigma(n, n-1)}{\Sigma(n-1, n-1)}
$$

and subtracting it from $(n-1)$ th column and using the determinant rule, we have

$$
\left|\mathbf{A}^{(n)}\right|=\left|\begin{array}{ccccc}
\cdot & \cdots & . & \cdot & 0 \\
\vdots & \vdots & \vdots & \vdots & \vdots \\
\cdot & \cdots & \cdot & \cdot & 0 \\
\cdot & \cdots & \cdot & A^{\prime}(n-1, n-1) & A(n-1, n) \\
0 & \cdots & 0 & 0 & A(n, n)
\end{array}\right|
$$

where

$$
\begin{aligned}
A^{\prime}(n-1, n-1):=A( & n-1, n-1) \\
& +\frac{\Sigma(n, n-1)}{\Sigma(n-1, n-1)} A(n, n-1) .
\end{aligned}
$$

Hence, we have

$$
\left|\mathbf{A}^{(n)}\right|=A(n, n)\left|\mathbf{M}_{n}\right|, \quad \text { for } n>1
$$

where $\mathbf{M}_{n}$ is the minor of $A(n, n)$ in (39). Substituting in (40), we have $A^{\prime}(n-1, n-1)=C$, where as noted before, $C$ is the contributions from nodes in $\mathcal{V}^{\prime} \backslash V_{n}$ and having an edge with $V_{n-1}$. This implies that $A^{\prime}(n-1, n-1)$ is the coefficient in the potential matrix for the subgraph induced by $\mathcal{V}^{\prime} \backslash V_{n}$. Since only $V_{n-1}$ has an edge with $V_{n}$, coefficients of nodes other than $V_{n}$ and $V_{n-1}$ are unaffected by the removal of $V_{n}$. Hence, $\mathbf{M}_{n}$ is the potential matrix for the subgraph induced by $\mathcal{V}^{\prime} \backslash V_{n}$,

$$
\mathbf{M}_{n}=\mathbf{A}^{(n-1)} .
$$

Since $\mathcal{V}^{\prime} \backslash V_{n}$ is acyclic, a leaf is always present, rearrange the rows such that $\mathbf{A}^{(n-1)}$ has a leaf in the last two rows, i.e., it has the same structure as in (39). Remove a leaf in each step of the recursion, until all the edges are removed, then find the determinant with the diagonal matrix consisting of the remaining nodes and we obtain (12).

\section{APPENDIX B \\ PROOF OF LEMMA 2}

We can regard $Y_{i}$ 's as marking, since under $\mathcal{H}_{0}$ they are i.i.d. independent of spatial point process. The strong stabilization condition is satisfied for NNG [4, Lemma 6.1, p. 1023]. We therefore only need to prove the uniform bounded moment condition. We express the edge functional as the sum of two functionals, for $i \sim j$, given by

$$
\begin{aligned}
& \phi_{1}\left(R_{i j}\right):=-\log \left[1-g^{2}\left(R_{i j}\right)\right] \\
& \phi_{2}\left(R_{i j}\right):=\frac{g^{2}\left(R_{i j}\right)\left[Y_{i}^{2}+Y_{j}^{2}\right]-2 g\left(R_{i j}\right) Y_{i} Y_{j}}{1-g^{2}\left(R_{i j}\right)} .
\end{aligned}
$$

Given a finite marked set $\mathcal{V}$, the sum functional is denoted by $H$ i.e.

$$
H_{k}(\mathcal{V}):=\sum_{\substack{(i, j) \in \mathrm{NNG}(\mathcal{V}) \\ i, j \in \mathcal{V}}} \frac{\phi_{k}\left(R_{i j}\right)}{2}, k=1,2 .
$$

Given $H_{k}$, we denote the add one cost [33, eq. (3.1)], which is the increment in $H$, caused by inserting a marked point at the origin into a finite marked set $\mathcal{V}$, by

$$
\Delta_{k}(\mathcal{V}):=H_{k}(\mathcal{V} \cup\{\mathbf{0}\})-H_{k}(\mathcal{V}) .
$$

$H_{1}$ satisfies the polynomial-bounded condition [33, eq. (3.3)], since $\phi_{1}$ in (41) is a finite function, and the number of edges in NNG is at most $n-1$, for $n$ points. However, the functional $H_{2}$ does not satisfy the polynomial-bounded condition since the measurements $Y_{i}$ in (42) are unbounded. Instead, we define truncated random variable $Z$ as

$$
Z:= \begin{cases}Y, & \text { if }|Y| \leq C \log n \\ \operatorname{sgn}(Y) C \log n, & \text { o.w. }\end{cases}
$$

where sgn is the sign function and $C>0$ is a constant. Consider the functionals $H_{2}^{\prime}, \phi_{2}^{\prime}$ by replacing $\mathbf{Y}_{n}$ with $\mathbf{Z}_{n}$ in $H_{2}$ and 
$\phi_{2}$ respectively. Now, $H_{2}^{\prime}$ is polynomially bounded. Further, we have $\lim _{n \rightarrow \infty} Z \stackrel{a . s .}{\rightarrow} Y$ and hence, $\lim _{n \rightarrow \infty}\left(H_{2}^{\prime}-H_{2}\right) \stackrel{a . s .}{\rightarrow} 0$.

Definition 3 (Uniform Bounded Moments for $\phi_{i}$ ): Define $\mathcal{U}_{m, A}$ to be $m$ uniform random variables on $A \in \mathcal{B}$ and $R_{\mathbf{0}, \mathbf{X}}$ to be the (random) lengths of the edge $(\mathbf{0}, \mathbf{X})$ in graph $\mathcal{G}$ incident on the origin. Then, the bounded $p$-moment condition [33, eq. (3.7)]

$$
\begin{aligned}
\sup _{A \in \mathcal{B}, 0 \in A} \sup _{m \in\left[\frac{\lambda|A|}{2}, \frac{3 \lambda|A|}{2}\right]} \mathbb{E}\left[\sum_{\substack{(\mathbf{0}, \mathbf{X}) \in \mathcal{G} \\
\mathbf{X} \in \mathcal{U}_{m}, A}} \phi_{k}\left(R_{\mathbf{0}, \mathbf{X}}\right)\right]^{p}<\infty, \\
\quad k=1,2
\end{aligned}
$$

is true for some $p \geq 1$.

Without the above condition, nothing can be said about the almost sure convergence, although, by Fatou's lemma, the limit of the LLN would be a bound on $D$.

Since $\phi_{1}$ and $\phi_{2}$ are decreasing functions edge length, with maximum at zero, we have

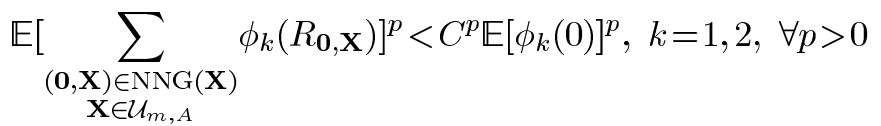

where $C$ is the kissing number, a constant, and $\operatorname{Deg}(0) \leq C$ for the NNG. Now, $\phi_{1}(0)=-\log \left[1-M^{2}\right]<\infty$, since $g(0)=$ $M<1$, and

$$
\mathbb{E}\left[\phi_{2}(0)^{p}\right]<\frac{M^{p}}{\left(1-M^{2}\right)^{p}} \mathbb{E}\left[Y_{i}-Y_{j}\right]^{2 p}<\infty
$$

since $Y_{i}, Y_{j} \stackrel{\text { i.i.d. }}{\sim} \mathcal{N}\left(0, \sigma_{0}^{2}\right)$. Hence, the uniform-bounded moment for $\phi_{k}$ in (46) holds.

Now, we show the uniform-bounded moment for $H$ [33, eq. (3.2)], obtained by replacing $\phi_{k}$ in (46) by $\Delta_{k}$. The positive part of $\Delta_{k}$ is bounded by $\operatorname{Deg}(0) \phi_{k}(0)$, whose expectation is shown to be finite. For the negative part $\Delta_{k}\left(\mathcal{U}_{m, \mathcal{A}}\right)^{-}$, along the lines of [4, Lemma 6.2], let $1\{n n(i)=0\}$ be the event that the origin is the nearest neighbor of $i \in \mathcal{U}_{m, \mathcal{A}}$. Then, the number of deleted edges on adding the origin is given by $\sum_{i=1}^{m} 1\{\mathrm{nn}(i)=$ $0\} \leq C$, we have $\Delta_{k}\left(\mathcal{U}_{m, \mathcal{A}}\right)^{-} \leq C \phi_{k}(0)$, whose expectation is shown to be finite. Hence, the bounded-moment condition for $H$ holds and LLN is applicable.

\section{APPENDIX C \\ PROOF OF THEOREM 3}

We have the distribution of $\mathbf{Y}_{n}$ under the null hypothesis

$$
p\left[\mathbf{Y}_{n} \mid \mathcal{V} ; \mathcal{H}_{0}\right]=\frac{1}{\left(2 \pi \sigma_{0}^{2}\right)^{\frac{n}{2}}} \exp \left(-\frac{\sum_{i=1}^{n} Y_{i}^{2}}{2 \sigma_{0}^{2}}\right)
$$

Therefore, the limit of the determinant is given by

$$
\lim _{n \rightarrow \infty} \frac{\log \left|\Sigma_{0, \mathcal{V}}\right|}{2 n}=\log \sigma_{0} \text {. }
$$

We have $\sum_{i=1}^{n} \frac{Y_{i}^{2}}{n} \rightarrow \mathbb{E}\left[Y_{1}^{2} ; \mathcal{H}_{0}\right]=\sigma_{0}^{2}$ a.s. under $\mathcal{H}_{0}$. Therefore, the term in (24)

$$
\sum_{i=1}^{n}\left(\frac{1}{\sigma_{1}^{2}}-\frac{1}{\sigma_{0}^{2}}\right) \frac{Y_{i}^{2}}{n} \rightarrow\left(\frac{\sigma_{0}}{\sigma_{1}}\right)^{2}-1
$$

By Lemma 2, the conditions for LLN hold and therefore as $n \rightarrow \infty$

$$
\begin{aligned}
& -\frac{1}{n} \sum_{\substack{e \in \mathrm{NNG}(\mathcal{V}) \\
\rightarrow-\mathbb{E}} \sum_{\substack{\mathbf{X}: \mathbf{X} \in \mathcal{P}_{\lambda} \\
(\mathbf{0}, \mathbf{X}) \in \operatorname{NNG}(\mathbf{X})}} \log \left[1-g^{2}\left(R_{e}\right)\right]} \log \left[1-g^{2}\left(R_{\mathbf{0}, \mathbf{X}}\right)\right]
\end{aligned}
$$

$$
\begin{aligned}
& \frac{1}{n} \sum_{\substack{(i, j) \in \mathrm{NNG}(\mathcal{V}) \\
i<j}} \frac{g^{2}\left(R_{i j}\right)\left[Y_{i}^{2}+Y_{j}^{2}\right]-2 g\left(R_{i j}\right) Y_{i} Y_{j}}{\left[1-g^{2}\left(R_{i j}\right)\right] \sigma_{1}^{2}} \\
& \rightarrow \mathbb{E} \sum_{\substack{\mathbf{X}: \mathbf{X} \in \mathcal{P}_{\lambda} \\
(\mathbf{0}, \mathbf{X}) \in \mathrm{NNG}(\mathbf{X})}} \frac{2 g^{2}\left(R_{\mathbf{0}, \mathbf{X}}\right)}{1-g^{2}\left(R_{\mathbf{0}, \mathbf{X}}\right)}\left(\frac{\sigma_{0}}{\sigma_{1}}\right)^{2}
\end{aligned}
$$

where, in (49) we first take the expectation over $Y_{i}$ 's and use the fact that $\mathbb{E}\left[\frac{Y_{\mathbf{0}}^{2}+Y_{\mathbf{X}}^{2}}{\sigma_{1}^{2}}\right]=2\left(\frac{\sigma_{0}}{\sigma_{1}}\right)^{2}$ and $\mathbb{E}\left[Y_{\mathbf{0}} Y_{\mathbf{X}}\right]=0$. Collecting all the terms we have (25).

\section{APPENDIX D \\ PROOF OF LEMMA 3}

We use an approach similar to [47]. Let $B_{z}(\mathbf{X})$ denote a circle of radius $z$, centered at $\mathbf{X}$. We take expectation on both sides of (27) for graphs over all the Poisson points $\mathbf{X} \cup \mathbf{0}$. Let $\mathrm{NNG}(\mathcal{V}), \mathrm{DNNG}$ and MNNG $\subset \mathrm{DNNG}$ be the undirected nearest-neighbot graph, the directed nearest-neighbor graph, and edges between the biroots or the mutual neighbors of the directed nearest-neighbor graph. See Fig. 3.

$$
\begin{aligned}
& \mathbb{E}\left[\sum_{\mathbf{X}: \mathbf{X} \in \mathcal{P}_{\lambda}} f\left(g\left(R_{\mathbf{0}, \mathbf{X}}\right)\right)\right]=\mathbb{E}\left[\sum_{\mathbf{X}: \mathbf{X} \in \mathcal{P}_{\lambda},} f\left(g\left(R_{\mathbf{0}, \mathbf{X}}\right)\right)\right] \\
& (\mathbf{0}, \mathbf{X}) \in \operatorname{NNG}(\mathbf{X}) \\
& (\mathbf{0}, \mathbf{X}) \in \operatorname{DNNG}(\mathbf{X}) \\
& -\frac{1}{2} \mathbb{E}\left[\sum_{\substack{\mathbf{X}: \mathbf{X} \in \mathcal{P}_{\lambda} \\
(\mathbf{0}, \mathbf{X}) \in \operatorname{MNNG}(\mathbf{X})}} f\left(g\left(R_{\mathbf{0}, \mathbf{X}}\right)\right)\right]
\end{aligned}
$$

The first term on the right-hand side in (50) simplifies as

$$
\mathbb{E}\left[\sum_{\substack{\mathbf{X}: \mathbf{X} \in \mathcal{P}_{\lambda},(\mathbf{0}, \mathbf{X}) \in \operatorname{DNNG}(\mathbf{X})}} f\left(g\left(R_{\mathbf{0}, \mathbf{X}}\right)\right)\right]=\mathbb{E}\left[f\left(g\left(Z_{1}\right)\right)\right]
$$

where $Z_{1}$ is the unique directed nearest-neighbor distance of the origin with points distributed according to $\mathcal{P}_{\lambda}$, the Poisson point process of intensity $\lambda$ on $\Re^{2}$. The random variable $Z_{1}$ is like a waiting time, and can be visualized as the time taken for an inflating circle to first touch a point from the Poisson process. We therefore have $Z_{1}>z$ if and only if (iff). $B_{z}(\mathbf{0})$ does not contain any points from the Poisson process, i.e.

$$
\mathbb{P}\left[Z_{1}>z\right]=\mathbb{P}\left[\nexists \mathbf{X} \neq \mathbf{0} \in B_{z}(\mathbf{0}) \cap \mathcal{P}_{\lambda}\right]=\mathrm{e}^{-\lambda \pi z^{2}} .
$$

Therefore, $Z_{1}$ is Rayleigh with second moment $(2 \pi \lambda)^{-1}$.

Similarly, for the second term, we need to find the PDF of the nearest-neighbor distance of the origin when the origin is a biroot or a mutual nearest neighbor. This event occurs when the union of the circles centered at origin and its nearest neighbor contains no other Poisson point. See Fig. 7. Let $\mathcal{A}$ be the intersection of the events that the directed nearest-neighbor distance 


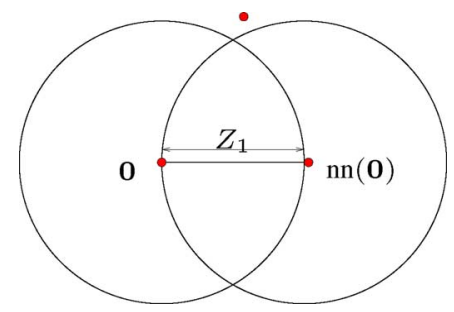

Fig. 7. Illustration of the event that the origin is a biroot in the directed NNG. This implies that there is no other point in the union of the circles shown above. See (53).

of origin lies in the interval $[z, z+d z]$ and the event that origin is a biroot

$$
\begin{aligned}
\mathcal{A}:= & \left(\mathcal{P}_{\lambda} \cap\left(B_{z}(\mathbf{0}) \cup B_{z}(\mathrm{nn}(\mathbf{0}))\right) \backslash\{\mathbf{0}, \mathrm{nn}(\mathbf{0})\}=\emptyset\right) \\
& \cap\left(Z_{1} \in[z, z+d z]\right) .
\end{aligned}
$$

Its probability is given by,

$$
\begin{aligned}
\mathbb{P}[\mathcal{A}] & =\mathbb{P}\left(\text { origin is biroot } \mid Z_{1}\right) \mathbb{P}\left(Z_{1} \in[z, z+d z]\right) \\
& =\mathrm{e}^{-(\omega-\pi) \lambda z^{2}} 2 \lambda \pi z \mathrm{e}^{-\lambda \pi z^{2}} d z \\
& =2 \lambda \pi z \mathrm{e}^{-\omega \lambda z^{2}} d z=\frac{\lambda}{\omega}\left[2 \omega \pi z \mathrm{e}^{-\omega \lambda z^{2}} d z\right] \\
& =\frac{\lambda}{\omega} \mathbb{P}\left(Z_{2} \in[z, z+d z]\right)
\end{aligned}
$$

where $\mathrm{nn}(\mathbf{0})$ is the nearest-neighbor of the origin and $\omega:=$ $\left|B_{1}(\mathbf{0}) \cup B_{1}(\mathbf{1})\right|=\frac{4 \pi}{3}+\frac{\sqrt{3}}{2}$, the area of the union of circles unit distant apart and $Z_{2}$ is a Rayleigh variable with variance $(2 \pi \omega)^{-1}$. Hence, the second term on the right-hand side in (50) simplifies as

$$
\frac{1}{2} \mathbb{E}\left[\sum_{\substack{\mathbf{X}: \mathbf{X} \in \mathcal{P}_{\lambda} \\(\mathbf{0}, \mathbf{X}) \in \mathrm{MNNG}(\mathbf{X})}} f\left(g\left(R_{\mathbf{0}, \mathbf{X}}\right)\right)\right]=\frac{\pi}{2 \omega} \mathbb{E}\left[f\left(g\left(Z_{2}\right)\right)\right] .
$$

From (27), (52), and (56), we obtain (28).

\section{ACKNOWLEDGMENT}

The authors thank Prof. J. E. Yukich for extensive discussions and clarifications regarding the law of large numbers for graph functionals. The authors also thank the anonymous reviewers and the Associate Editor Prof. A. Høst-Madsen for detailed comments that substantially improved this paper.

\section{REFERENCES}

[1] A. Anandkumar, L. Tong, and A. Swami, "Detection of Gauss-Markov random fields with nearest-neighbor dependency," in Proc. ICASSP'07, Honolulu, HI, Apr. 2007, vol. III, pp. 829-832.

[2] J. Moura and S. Goswami, "Gauss Markov random fields (GMrf) with continuous indices," IEEE Trans. Inf. Theory, vol. IT-43, no. 5, pp. 1560-1573, Sep. 1997.

[3] L. Devroye, "The expected size of some graphs in computational geometry," Comp. Math. App., vol. 15, no. 1, pp. 53-64, 1988.

[4] M. D. Penrose and J. E. Yukich, "Central limit theorems for some graphs in computational geometry," Ann. Appl. Prob., vol. 11, no. 4, pp. 1005-1041, 2001.

[5] J. Moura and N. Balram, "Recursive structure of noncausal GaussMarkov random Fields," IEEE Trans. Inf. Theory, vol. 38, no. 2, pp. 334-354, Mar. 1992.

[6] N. Cressie, Statistics for Spatial Data. New York: Wiley, 1993.

[7] R. Pace and D. Zou, "Closed-form maximum likelihood estimates of nearest neighbor spatial dependence," Geograph. Anal., vol. 32, no. 2, pp. 154-172, 2000.

[8] H. Rue and L. Held, Gaussian Markov Random Fields: Theory and Applications. London, U.K.: Chapman and Hall, 2005.

[9] R. Ahlswede and I. Csiszar, "Hypothesis testing with communication constraints," IEEE Trans. Inf. Theory, vol. 32, no. 4, pp. 533-542, 1986.
[10] T. S. Han and S. Amari, "Statistical inference under multiterminal data compression," IEEE Trans. Inf. Theory, vol. 44, no. 6, pp. 2300-2324, Oct. 1998.

[11] J. Besag, "Spatial interaction and the statistical analysis of lattice systems," J. Roy. Stat. Soc., vol. 36, no. B, pp. 192-225, 1974.

[12] J. Besag, "Statistical analysis of non-lattice data," The Statistician, vol. 24, no. 3, pp. 179-195, 1975.

[13] X. Guyon, Random Fields on a Network: Modeling, Statistics, and Applications. New York: Springer, 1995.

[14] T. Kailath and H. Poor, "Detection of stochastic processes," IEEE Trans. Inf. Theory, vol. 44, no. 6, pp. 2230-2259, Oct. 1998.

[15] M. D. Donsker and S. R. S. Varadhan, "Large deviations for stationary Gaussian process," Commun. Math. Phys., vol. 97, pp. 187-210, 1985.

[16] G. Benitz and J. Bucklew, "Large deviation rate calculations for nonlinear detectors in Gaussian noise," IEEE Trans. Inf. Theory, vol. 36, no. 2, pp. 358-371, Mar. 1990.

[17] R. Bahr, "Asymptotic analysis of error probabilities for the nonzeromean Gaussian hypothesis testing problem," IEEE Trans. Inf. Theory, vol. 36, no. 3, pp. 597-607, May 1990.

[18] R. Bahr and J. Bucklew, "Optimal sampling schemes for the Gaussian hypotheis testing problem," IEEE Trans. on Acoust., Speech, Signal Processing, vol. 38, no. 10, pp. 1677-1686, Oct. 1990.

[19] P. Barone, A. Gigli, and M. Piccioni, "Optimal importance sampling for some quadratic forms of ARMA processes," IEEE Trans. Inf. Theory, vol. 41, no. 6, pp. 1834-1844, Nov. 1995.

[20] W. Bryc and W. Smolenski, "On the large deviation principle for a quadratic functional of the autoregressive process," Statist. Probab. Lett., vol. 17, pp. 281-285, 1993.

[21] W. Bryc and A. Dembo, "Large deviations for quadratic functionals of Gaussian processes," J. Theoret. Probab., vol. 10, no. 2, pp. 307-332, 1997.

[22] B. Bercu, F. Gamboa, and A. Rouault, "Large deviations for quadratic forms of stationary Gaussian processes," Stoch. Process. Appl., vol. 71, pp. 75-90, 1997.

[23] B. Bercu and A. Rouault, "Sharp large deviations for the Ornstein-Uhlenbeck process," Theory Probab. Appl., vol. 46, no. 1, pp. 1-19, 2002.

[24] J.-F. Chamberland and V. V. Veeravalli, "Design of sensor networks for detection applications via large-deviation theory," in Proc. 2004 IEEE Information Theory Workshop, San Antonio, TX, Oct. 2004.

[25] P.-N. Chen, "General formulas for the Neyman-Pearson type-II error exponent subject to fixed and exponential type-I error bounds," IEEE Trans. Inf. Theory, vol. 42, no. 1, pp. 316-323, Jan. 1996.

[26] I. Vajda, "Distances and discrimination rates of stochastic processes," Stoch. Process. Appl., vol. 35, pp. 47-57, 1990.

[27] H. Luschgy, A. L. Rukhin, and I. Vajda, "Adaptive tests for stochastic processes in the ergodic case," Stoch. Process. Appl., vol. 45, pp. 47-59, 1990.

[28] Y. Sung, L. Tong, and H. Poor, "Neyman-Pearson detection of GaussMarkov signals in noise: Closed-form error exponent and properties," IEEE Trans. Inf. Theory, vol. 52, no. 4, pp. 1354-1365, Apr. 2006.

[29] A. Kavčić and M. Moura, "Matrices with banded inverses: Inversion algorithm and factorization of Gauss-Markov processes," IEEE Trans. Inf. Theory, vol. 46, no. 4, pp. 1495-1509, Jul. 2000.

[30] A. Asif and J. Moura, "Block matrices with L-block-banded inverse: Inversion algorithms," IEEE Trans. Signal Process., vol. 53, no. 2, pp. 630-642, Feb. 2005

[31] R. Cowell, Probabilistic Networks and Expert Systems. New York: Springer, 1999.

[32] M. Wainwright, T. Jaakkola, and A. Willsky, "Tree-based reparameterization framework for analysis of sum-product and related algorithms," IEEE Trans. Inf. Theory, vol. 49, no. 5, pp. 1120-1146, May 2003.

[33] M. D. Penrose and J. E. Yukich, "Limit theory for random sequential packing and deposition," Ann. Appl. Probab., vol. 12, no. 1, pp. 272-301, 2002.

[34] M. D. Penrose and J. E. Yukich, "Weak laws of large numbers in geometric probability," Ann. Appl. Probab., vol. 13, no. 1, pp. 277-303, 2003.

[35] J. Møller, Spatial Statistics and Computational Methods. New York: Springer, 2003.

[36] M. Jaksa, P. Brooker, and W. Kaggwa, "Inaccuracies associated with estimating random measurement errors," J. Geotechn. Geoenviron. Eng., vol. 123, no. 5, pp. 393-401, 2006.

[37] G. Grundmann and D. Debouzie, "Geostatistical analysis of the distribution of $\mathrm{NH} 4$ and $\mathrm{NO}$ 2-oxidizing bacteria and serotypes at the millimeter scale along a soil transect," FEMS Microbiol. Ecol., vol. 34, pp. 57-62, 2000.

[38] M. Barange and I. Hampton, "Spatial structure of co-occurring anchovy and sardine populations from acoustic data: Implications for survey design," Fisheries Oceanogr., vol. 6, no. 2, pp. 94-108, 1997.

[39] C. Zong, "The kissing numbers of convex bodies- A brief survey," Bull. London Math.Soc., vol. 30, no. 01, pp. 1-10, 2000.

[40] D. Eppstein, M. S. Paterson, and F. F. Yao, "On nearest-neighbor graphs,” Discr. Comput. Geometry, vol. 17, pp. 263-282, 1997. 
[41] A. Anandkumar, L. Tong, A. Swami, and A. Ephremides, "Minimum cost data aggregation with localized processing for statistical inference," in Proc. INFOCOM, Phoenix, AZ, Apr. 2008, pp. 780-788.

[42] T. Han, "Hypothesis testing with the general source," IEEE Trans. Inf. Theory, vol. 46, no. 7, pp. 2415-2427, Nov. 2000.

[43] T. Cover and J. Thomas, Elements of Information Theory. New York: Wiley, 1991.

[44] J. Møller and R. Waagepetersen, Statistical Inference and Simulation for Spatial Point Processes. London, U.K.: Chapman and Hall, 2004.

[45] J. Steele, Probability Theory and Combinatorial Optimization. Philadelphia, PA: Soc. Industr. Appl. Math., 1997.

[46] A. Anandkumar and L. Tong, "A large deviation analysis of detection over multi-access channels with random number of sensors," in Proc. ICASSP'06, Toulouse, France, May 2006, vol. IV, pp. 1097-1101.

[47] A. Wade, "Explicit laws of large numbers for random nearest-neighbour type graphs," Adv. Appl. Probab., vol. 39, 2007.

[48] A. Baddeley, "A crash course in stochastic geometry," in Stochastic Geometry: Likelihood and Computation, O. E. Barndorff-Nielsen, W. S. Kendall, and M. N. M. van Lieshout, Eds. London, U.K.: Chapman and Hall, 1999.

[49] H. Rue, I. Steinsland, and S. Erland, "Approximating hidden Gaussian Markov random fields," J. Roy. Stat. Soc. Series B (Stat. Methodol.), vol. 66, no. 4, pp. 877-892, 2004.

[50] H. Rue and H. Tjelmeland, "Fitting Gaussian Markov random fields to Gaussian Fields," Scand. J. Stat., vol. 29, no. 1, pp. 31-49, 2002.

[51] A. Anandkumar, L. Tong, and A. Swami, "Optimal mnode density for detection in energy-constrained random networks," IEEE Trans. Signal Process., vol. 56, no. 10, pp. 5232-5245, Oct. 2008.

[52] A. Anandkumar, L. Tong, A. Swami, and A. Ephremides, "Cost-performance tradeoff in multi-hop aggregation for statistical inference," in Proc. IEEE ISIT, Toronto, ON, Canada, Jul. 2008, pp. 662-666.

Animashree Anandkumar ( $\mathrm{S}^{\prime}$ '02) received the B.Tech. degree in electrical engineering from Indian Institute of Technology Madras, Chennai, India, in 2004. She is currently pursuing the Ph.D. degree in electrical engineering at Cornell University, Ithaca, NY.

She has been a member of the Adaptive Communications and Signal Processing Group (ACSP) at Cornell University since August, 2004. Her research interests are in the area of statistical-signal processing, information theory and networking. Specifically, she has been working on detection and estimation, asymptotic analysis and in-network function computation, in the context of wireless-sensor networks. She has served as a reviewer for IEEE TRAnsactions on Signal Processing, IEEE TRANSACtions ON INFORMATION THEORY, and various IEEE conferences.

Dr. Anandkumar received the Fran Allen IBM Ph.D. fellowship for the year 2008-2009, presented annually to one female Ph.D. student in conjunction with the IBM Ph.D. Fellowship Award. She was named a finalist for the Google Anita-Borg Scholarship 2007-2008. She received the Student Paper Award at the 2006 International Conference on Acoustic, Speech and Signal Processing (ICASSP) held at Toulouse, France.
Lang Tong (S'87-M'91-SM'01-F'05) received the B.E. degree from Tsinghua University, Beijing, China, in 1985, and the M.S. and Ph.D. degrees in electrical engineering in 1987 and 1991, respectively, from the University of Notre Dame, Notre Dame, IN.

He was a Postdoctoral Research Affiliate at the Information Systems Laboratory, Stanford University, Stanford, CA, in 1991. He is now the Irwin and Joan Jacobs Professor in Engineering at Cornell University Ithaca, NY. Prior to joining Cornell University, he was on faculty at the West Virginia University and the University of Connecticut. He was also the 2001 Cor Wit Visiting Professor at the Delft University of Technology. His research is in the general area of statistical signal processing, wireless communications and networking, and information theory.

Dr. Tong received the 1993 Outstanding Young Author Award from the IEEE Circuits and Systems Society, the 2004 best paper award (with Min Dong) from the IEEE Signal Processing Society, and the 2004 Leonard G. Abraham Prize Paper Award from the IEEE Communications Society (with Parvathinathan Venkitasubramaniam and Srihari Adireddy). He is also a coauthor of five student paper awards. He received Young Investigator Award from the Office of Naval Research. He has served as an Associate Editor for the IEEE TRANSACtions on Signal Processing, the IEEE TRANSACtions ON INFORMATION THEORY, and the IEEE SIGNAL PROCESSING LETTERS.

Ananthram Swami (F'07) received the B.Tech. degree from the Indian Institute of Technology-Bombay, India, the M.S. degree from Rice University, Houston, TX, and the Ph.D. degree from the University of Southern California (USC), Los Angeles, all in electrical engineering.

He has held positions with Unocal Corporation, USC, CS-3, and Malgudi Systems. He was a Statistical Consultant to the California Lottery, developed a Matlab-based toolbox for non-Gaussian signal processing, and has held visiting faculty positions at INP, Toulouse, France. He is currently with the US Army Research Laboratory where his work is in the broad area of signal processing, wireless communications, sensor and mobile ad hoc networks.

Dr. Swami has served as Associate Editor of the IEEE TRANSACTIONS on Wireless Communications, the IEEE Signal PROCESSING LetTers, the IEEE TRansactions on Circuits and Systems-II, the IEEE Signal PROCESSING MAGAZINE, and the IEEE TRANSACTIONS ON Signal PROCESSING. $\mathrm{He}$ was Co-Guest Editor of a 2004 Special Issue (SI) of the IEEE SIGNAL PROCESSING MAGAZINE (SPM) on Signal Processing for Networking, a 2006 SPM SI on Distributed Signal Processing in Sensor Networks, a 2006 EURASIP JASP SI on Reliable Communications Over Rapidly Time-Varying Channels, a 2007 EURASIP JWCN SI on Wireless Mobile Ad Hoc Networks, and is the Lead Editor for a 2008 IEEE JSTSP SI on "Signal Processing and Networking for Dynamic Spectrum Access." He is a Co-Editor, with Qing Zhao, Yao-Win Hong, and Lang Tong, of the book Wireless Sensor Networks: Signal Processing and Communications Perspectives (New York: Wiley, 2007). 